\title{
Análise de Risco das Mudanças Climáticas Globais sobre a Sigatoka-negra da Bananeira no Brasil
}

\author{
Raquel Ghini ${ }^{1}$, Emilia Hamada ${ }^{1}$, Renata R.V. Gonçalves ${ }^{1}$, \\ Luadir Gasparotto ${ }^{2}$ \& José Clério R. Pereira ${ }^{2}$
}

'Embrapa Meio Ambiente, Cx. Postal 69, CEP 13820-000, Jaguariúna, SP, Brasil, e-mail: raquel@cnpma.embrapa.br; ${ }^{2}$ Embrapa Amazônia Ocidental, Cx. Postal 319, CEP 69011-970 Manaus, AM, Brasil

Autor para correspondência: Raquel Ghini

GHINI, R., HAMADA, E., GONÇALVES, R.R.V., GASPAROTTO, L. \& PEREIRA, J.C.R. Análise de risco das mudanças climáticas globais sobre a sigatoka-negra da bananeira no Brasil. Fitopatologia Brasileira 32:197-204. 2007.

\section{RESUMO}

O conhecimento dos prováveis impactos das mudanças climáticas globais sobre a ocorrência de doenças de plantas é de grande importância para o setor agrícola, pois permite a elaboração de estratégias de controle. O presente trabalho teve por finalidade estudar os possíveis impactos das mudanças climáticas sobre a sigatoka-negra da bananeira, por meio da elaboração de mapas de distribuição da doença confeccionados a partir dos cenários disponibilizados pelo IPCC. Os mapas mostraram que haverá redução da área favorável à doença no país. Tal redução será gradativa para as décadas de 2020,2050 e 2080 e de forma mais acentuada no cenário A2 que no B2. Apesar disso, extensas áreas ainda continuarão favoráveis à ocorrência da doença, especialmente no período de novembro a abril.

Palavras-chave adicionais: Mycosphaerella fijiensis, Musa spp., banana, plátano.

\begin{abstract}
Risk analysis of climatic change on black Sigatoka on banana in Brazil

The knowledge of the probable impacts of global climatic changes on the occurrence of plant diseases is of great importance for the agricultural sector, since it allows the elaboration of control strategies. The aim of the present paper was to study the probable impacts of climatic changes on black Sigatoka disease of banana, by means of the elaboration of distribution maps of the disease using IPCC scenarios A2 and B2. The maps evidenced that there will be a reduction of the favorable area to the disease in the country. Such reduction will be gradual for the decades of 2020, 2050 and 2080 and higher for A2 scenario than for B2. Despite this, extensive areas will still continue favorable to the occurrence of the disease, especially in the period of November to April.
\end{abstract}

Additional keywords: Mycosphaerella fijiensis, Musa spp., banana, plantain.

\section{INTRODUÇÃO}

Nas últimas décadas, mudanças climáticas globais têm despertado as atenções de diversos segmentos da sociedade. $\mathrm{O}$ aumento da concentração de gases de efeito estufa resultante da intensificação das atividades antrópicas tem causado significativas alterações no clima (IPCC, 2007). A temperatura da superfície do planeta aumentou, aproximadamente, $0,2{ }^{\circ} \mathrm{C}$ por década nas últimas três décadas, e $0,8^{\circ} \mathrm{C}$ no século passado. Durante os primeiros cinco anos do século XXI, mapas de anomalias de temperatura em relação ao período de 1951-1980 demonstram que houve, de modo geral, aquecimento no planeta, porém o aumento de temperatura foi maior nos continentes que nos oceanos, e também nas maiores latitudes do Hemisfério Norte (Hansen et al., 2006). Além do aquecimento global, tem sido observada maior freqüência e intensidade de eventos climáticos extremos, alterações nos regimes de chuva, perturbações nas correntes marinhas, retração de geleiras e elevação do nível dos oceanos (Marengo, 2001; NAE, 2005a). Tais alterações podem resultar em graves problemas sociais, econômicos e ambientais. Dentre esses, a distribuição geográfica, a época de ocorrência e a severidade de doenças de plantas que podem ser alteradas (Ghini, 2005).

A Organização Meteorológica Mundial (OMM) e o Programa das Nações Unidas para o Meio Ambiente (PNUMA) criaram o Painel Intergovernamental sobre Mudanças Climáticas (IPCC) em 1988, com a finalidade de realizar análises sistemáticas sobre o conhecimento científico existente sobre as mudanças climáticas globais, seus impactos potenciais e as opções de mitigação e adaptação. Uma de suas mais importantes realizações foi a divulgação de um conjunto de cenários de emissão de gases de efeito estufa, que levam em consideração forçantes controladoras, como demografia, desenvolvimento sócio-econômico, mudança tecnológica, bem como suas interações. Assim, foram definidas quatro famílias de cenários: A1, A2, B1 e B2, que constituem referências com relação às emissões futuras de gases de efeito estufa (IPCC, 2001).

O Centro de Distribuição de Dados (DDC) do IPCC foi estabelecido para facilitar a divulgação desses cenários, assim como de fatores ambientais e sócio-econômicos a eles relacionados, para uso em avaliações dos impactos do 
clima. O IPCC-DDC permite acesso a resultados de modelos dos principais centros de modelagem do clima (Hadley Centre for Climate Prediction and Research da Inglaterra, Max Planck Institut für Meteorologie da Alemanha, Canadian Centre for Climate Modelling and Analysis do Canadá, Geophysical Fluids Dynamics Laboratory dos EUA, Commonwealth Scientific and Industrial Research Organization da Austrália, National Institute for Environmental Studies e Centre for Climate System Research do Japão). Esses modelos climáticos globais quantificam o comportamento dos compartimentos climáticos (atmosfera, oceanos, vegetação, solos, etc.) e suas interações, permitindo estimar as variáveis climáticas (temperatura média, máxima, mínima, precipitação, etc.) para os diversos cenários de emissões de gases de efeito estufa estabelecidos pelo IPCC (2001). As informações podem ser utilizadas livremente para a realização de trabalhos de pesquisa.

Os dados, entretanto, necessitam de cuidadoso tratamento de georeferenciamento para permitir o estudo de avaliação de impactos. Os resultados dos diversos modelos estão apresentados em diferentes grades, o que exige a realização de interpolações. Além disso, há diversas fontes de incertezas, como a baixa resolução temporal e espacial; a dificuldade em simular adequadamente os processos climáticos, devido à sua complexidade; e a dificuldade em estimar a exata quantidade de gases de efeito estufa que será liberada nas próximas décadas. Não obstante as dificuldades, trata-se da melhor ferramenta para projetar cenários prováveis de alterações climáticas para o futuro com a finalidade de estudo da vulnerabilidade do setor agrícola.

Dentre os problemas fitossanitários do país, a sigatokanegra, causada por Mycosphaerella fijiensis Morelet, constituise no principal fator de queda na produtividade dos bananais, com reduções de até $100 \%$ na produção comercial de bananas das cultivares suscetíveis, como 'Prata', 'Maçã', 'D'Angola' e as do sub-grupo Cavendish. Na região Amazônica, onde a umidade e a temperatura são altamente favoráveis, a sigatokanegra ocorre de forma severa o ano todo (Gasparotto et al., 2006). Devido à importância da doença, o presente trabalho teve como objetivo avaliar os possíveis impactos das mudanças climáticas globais sobre a sigatoka-negra da bananeira no Brasil, por meio da elaboração de mapas de distribuição da doença confeccionados a partir dos cenários disponibilizados pelo IPCC.

\section{MATERIAL E MÉTODOS}

Médias mensais de temperatura média e umidade relativa dos climas presente e futuros para o Brasil foram obtidas na página da internet do IPCC (2006). Para dados de temperatura, foram utilizados seis modelos: CCSR/NIES ("Centre for Climate Research Studies Model"), CGCM2 ("Canadian Global Coupled Model version 2"), CSIROMk2 ("Commonwealth Scientific and Industrial Research Organization GCM mark 2"), ECHAM4 ("European Centre Hamburg Model version 4"), GFDL-R30 (“Geophysical Fluid Dynamics Laboratory, R-30 resolution model”) e HadCM3
(“Hadley Centre Coupled Model version 3") (IPCC, 2006). Dados de umidade relativa estão disponibilizados somente pelo modelo HadCM3, assim sendo, para essa variável climática foram usados dados originados de um único modelo.

Para a caracterização do clima atual foram consideradas informações referentes ao período de 1961 a 1990. Os cenários futuros utilizados foram A2 e B2, centrados nas décadas de 2020 (período entre 2010 e 2039), 2050 (período entre 2040 e 2069) e 2080 (período entre 2070 e 2099) (IPCC, 2001).

O cenário A2 é um cenário de elevadas emissões de gases de efeito estufa, isto é, assume a manutenção dos padrões atuais de emissões. Descreve um mundo futuro muito heterogêneo, com preservação das identidades locais e da tradição. Os padrões de fertilidade entre regiões convergem muito lentamente, o que resulta em alto crescimento populacional. O desenvolvimento econômico é principalmente orientado regionalmente e o crescimento econômico per capita e a mudança tecnológica são mais fragmentados e mais lentos, comparados às outras famílias de cenário (IPCC, 2001).

$\mathrm{O}$ cenário B2 é um cenário de menores emissões, com características mais otimistas em relação ao cenário A2. Descreve um mundo no qual a ênfase está em soluções locais para a sustentabilidade econômica, social e ambiental. É um mundo com moderado crescimento populacional, níveis intermediários de desenvolvimento econômico e mudança tecnológica menos rápida e mais diversa, do que o B1 e o A1. É orientado para a proteção do meio ambiente e a eqüidade social, mas com foco nos níveis local e regional (IPCC, 2001).

O SIG (Sistema de Informações Geográficas) Idrisi 32 foi utilizado para a elaboração dos mapas. Devido às diferentes resoluções espaciais dos modelos disponibilizados pelo IPCC (CCSR/NIES: 5,625 X 5,625 $5^{\circ}$ CGCM: $3,75^{\circ} \mathrm{X}$ $3,75^{\circ}$, CSIRO-Mk2b: $5,625^{\circ}$ X 3,214 ${ }^{\circ}$, ECHAM4: $2,8125^{\circ}$

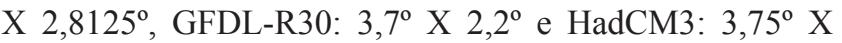
$2,5^{\circ}$ ), os dados foram interpolados pelo método de Inverso do Quadrado da Distância, de forma a terem a resolução espacial final de $0,5^{\circ} \mathrm{X} 0,5^{\circ}$ de latitude e longitude. A seguir, a área correspondente ao Brasil foi recortada dos dados georeferenciados e uma máscara delimitando os estados foi aplicada sobre os mapas.

A temperatura média mensal do cenário futuro foi obtida pela média dos seis modelos, utilizando as ferramentas de análise espacial do SIG (operação aritmética). A partir dos mapas de temperatura média e umidade relativa mensais, para os cenários $\mathrm{A} 2$ e $\mathrm{B} 2$, para o período atual e futuro (2020, 2050 e 2080), foram obtidos mapas apresentando as áreas favoráveis ou não ao desenvolvimento da doença. Para tanto, foi considerado que o desenvolvimento da doença é favorecido por temperaturas entre $20^{\circ} \mathrm{C}$ e $30^{\circ} \mathrm{C}$, e umidade relativa acima de 70 \% (Jacome \& Schuh, 1992; Mouliom-Pefoura et al., 1996; Romero \& Sutton, 1997). Conseqüentemente, regiões que apresentam temperaturas médias inferiores a $20{ }^{\circ} \mathrm{C}$ ou superiores a $30{ }^{\circ} \mathrm{C}$ ou umidade relativa média inferior a $70 \%$ foram consideradas desfavoráveis à doença. A seguir, as áreas favoráveis à doença foram calculadas em cada período estudado. 


\section{RESULTADOS E DISCUSSÃO}

Os mapas de favorabilidade climática à sigatokanegra da bananeira confeccionados para os cenários futuros indicam que, de um modo geral, haverá redução da área favorável ao desenvolvimento da doença no país, em relação ao clima atual, tanto para o cenário A2 (Figuras 1 e 2) quanto para o B2 (Figuras 3 e 4). Tal redução está projetada tanto para o período de maior favorabilidade à doença (meses

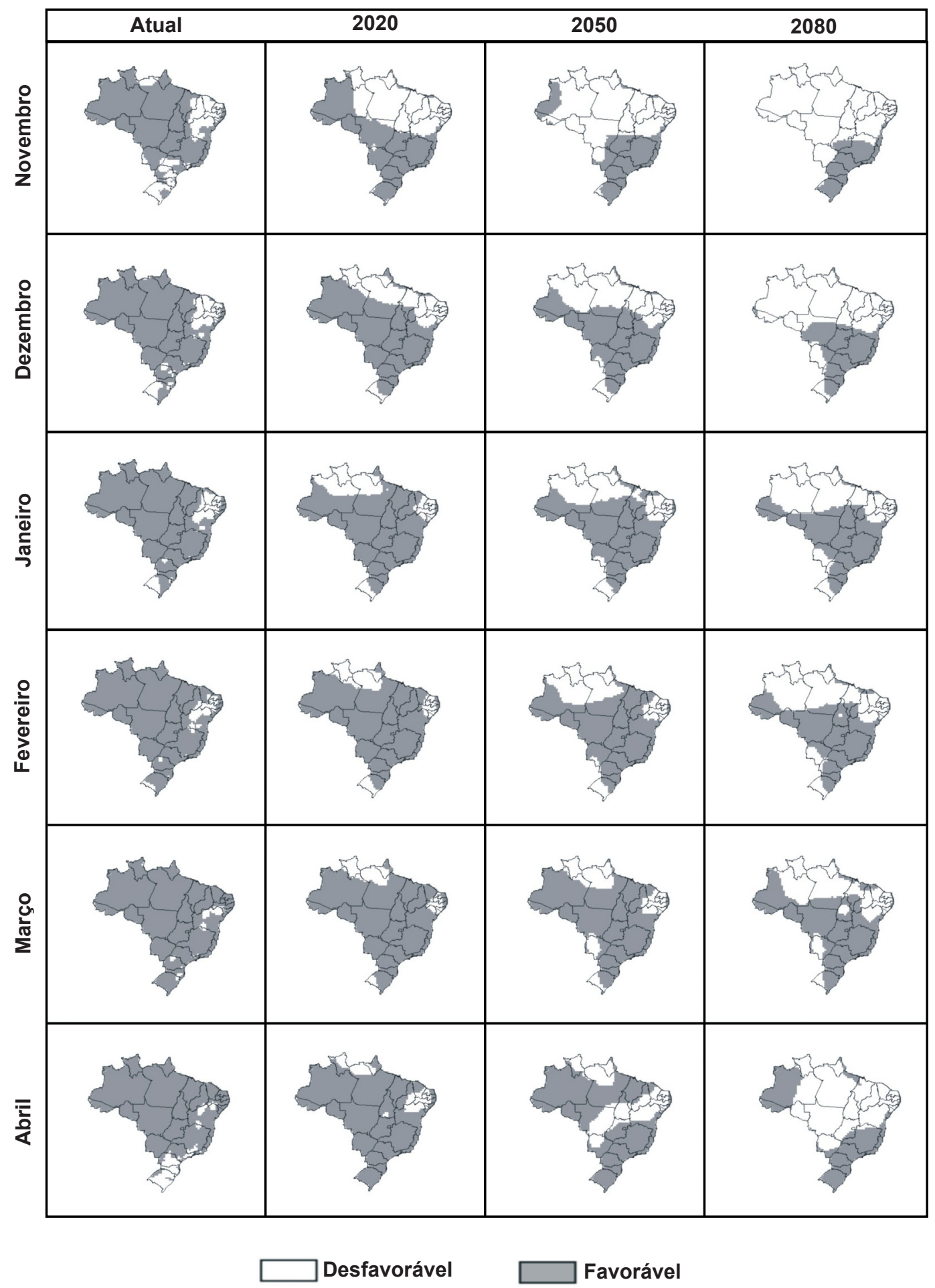

FIG. 1 - Mapa de favorabilidade climática à sigatoka-negra da bananeira no Brasil, para os meses de novembro a abril, no período atual (média de 1961 a 1990) e futuro (2020, 2050 e 2080, para o cenário A2). 


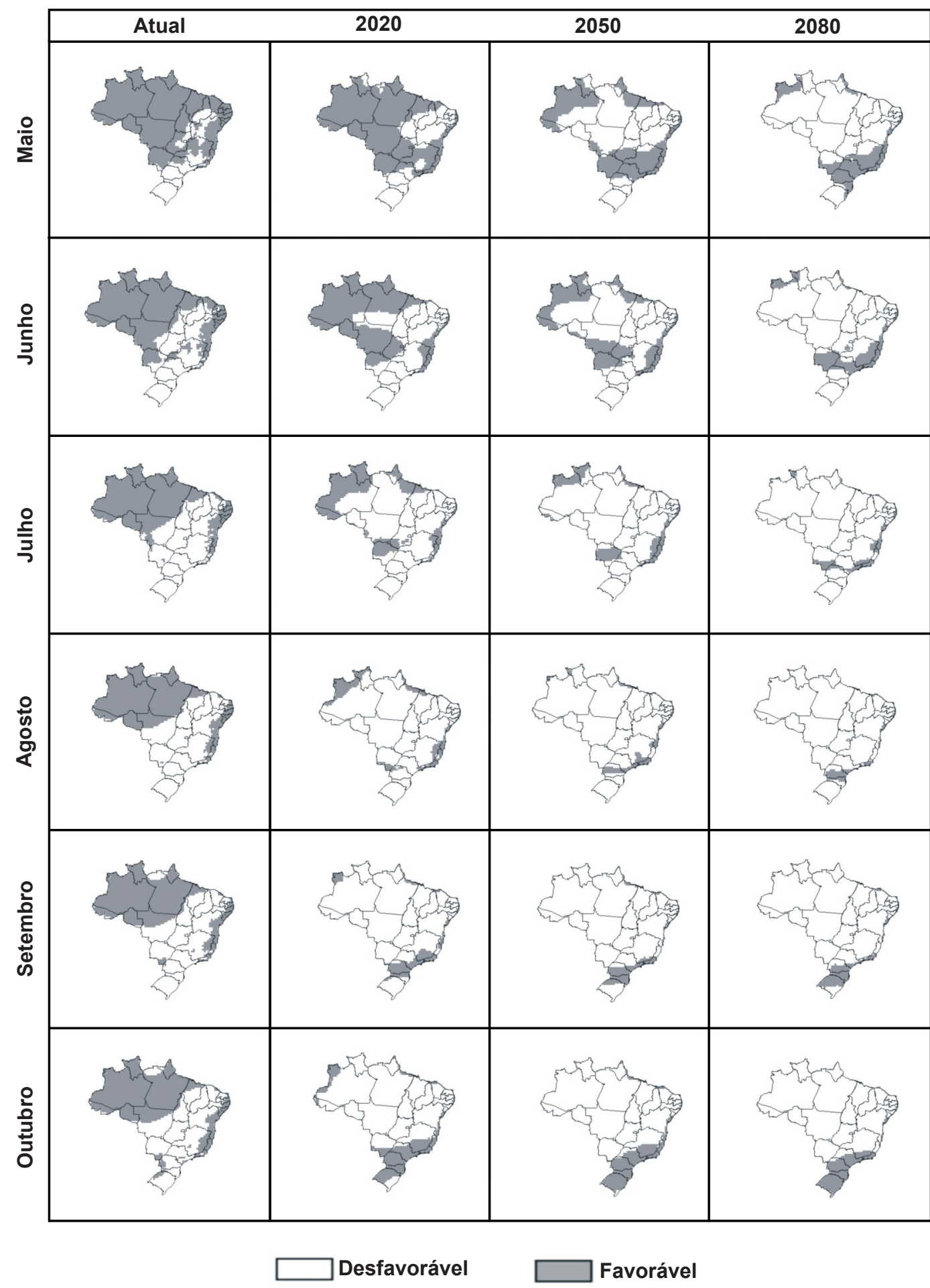

FIG. 2 - Mapa de favorabilidade climática à sigatoka-negra da bananeira no Brasil, para os meses de maio a outubro, no período atual (média de 1961 a 1990) e futuro (2020, 2050 e 2080, para o cenário A2).

de novembro a abril; Figuras 1 e 3 para os cenários A2 e $\mathrm{B} 2$, respectivamente), assim como para o período menos favorável (meses de maio a outubro; Figuras 2 e 4 para os cenários $\mathrm{A} 2$ e B2, respectivamente). A principal alteração do clima responsável por esse resultado é a redução da umidade relativa média para níveis desfavoráveis à ocorrência da doença, ou seja, valores abaixo de $70 \%$.

As reduções na incidência da doença serão mais acentuadas para o cenário A2 que para o B2 (Tabela 1). $\mathrm{O}$ cenário $\mathrm{A} 2$ prevê maiores reduções de umidade que $\mathrm{o}$ 


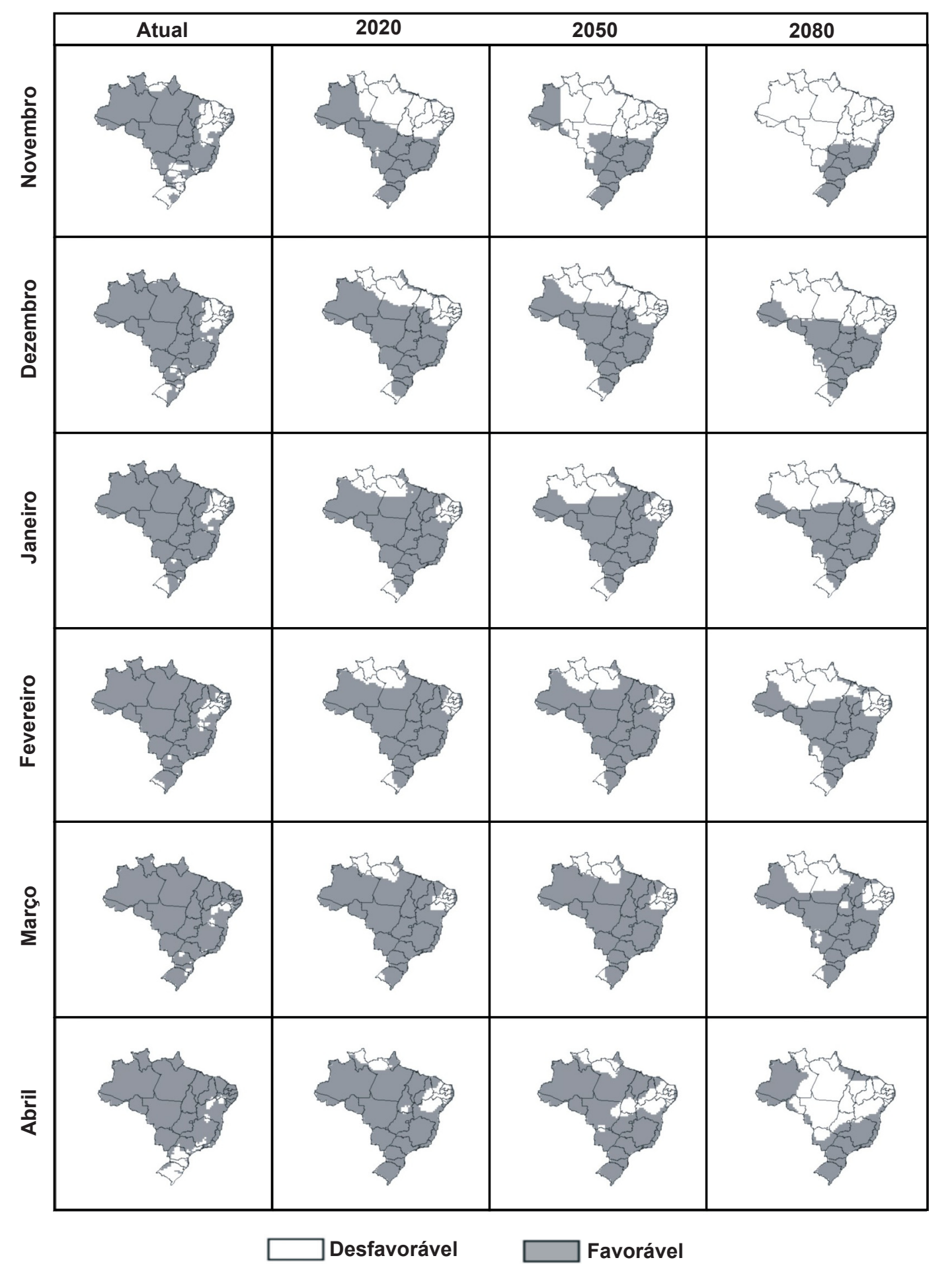

FIG. 3 - Mapa de favorabilidade climática à sigatoka-negra da bananeira no Brasil, para os meses de novembro a abril, no período atual (média de 1961 a 1990) e futuro (2020, 2050 e 2080, para o cenário B2).

cenário B2, resultando em condições menos favoráveis à sigatoka. Além disso, a redução da área favorável à doença é gradativa nas três décadas estudadas para os dois cenários, isto é, a cada década, a área favorável sofre reduções. Por exemplo, no período considerado atual, para os meses de novembro a abril, em média, a área favorável à sigatoka corresponde a $91 \%$ do território nacional e, para os meses de maio a outubro, $62 \%$. Em 2020, para o cenário A2, a área média será de $79 \%$ e $33 \%$; para 2050 será de $64 \%$ e $19 \%$ e para 2080 será de $43 \%$ e $10 \%$, respectivamente. 


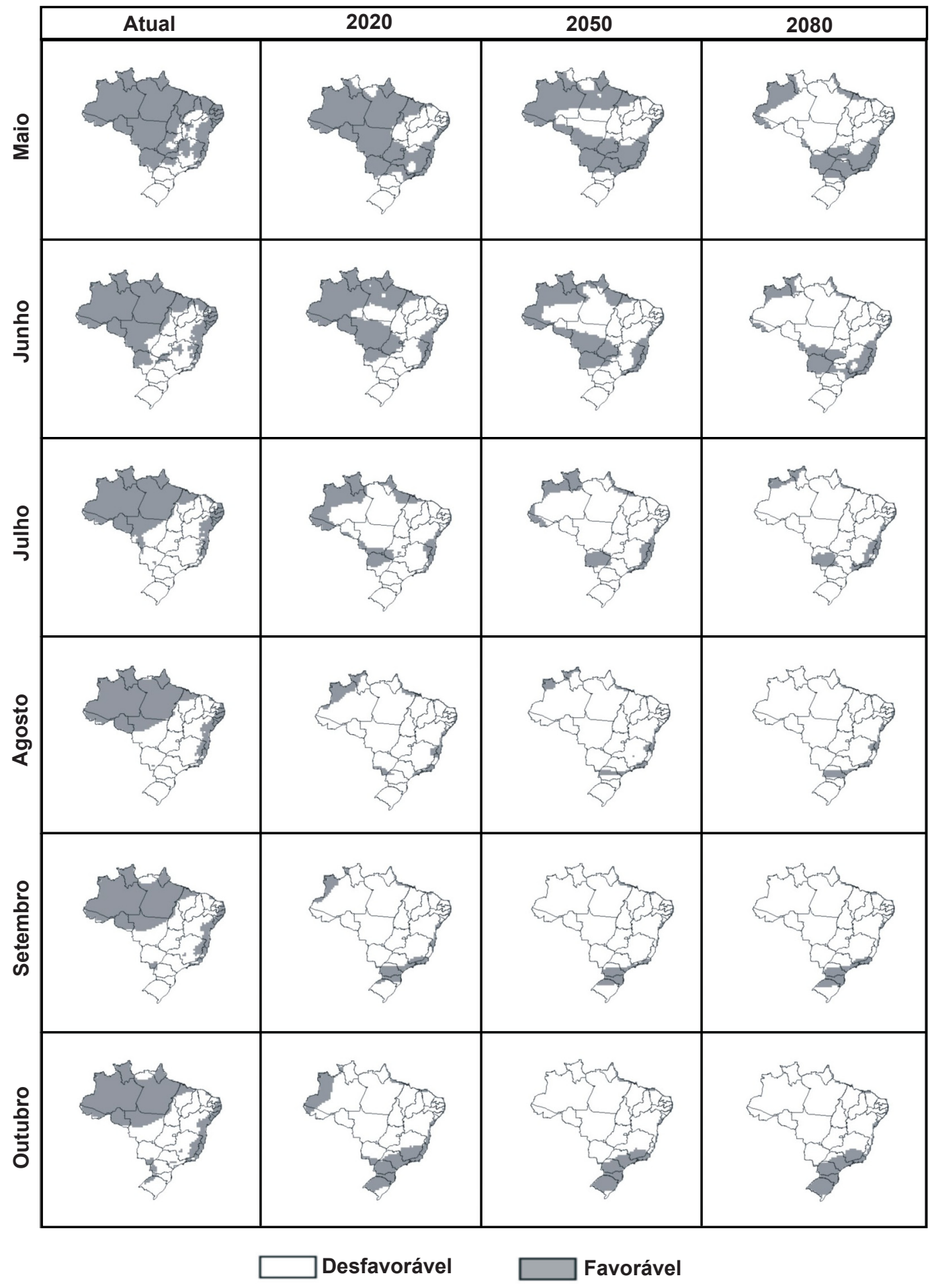

FIG. 4 - Mapa de favorabilidade climática à sigatoka-negra da bananeira no Brasil, para os meses de maio a outubro, no período atual (média de 1961 a 1990) e futuro (2020, 2050 e 2080, para o cenário B2).

Apesar de ocorrer redução, nota-se que extensas áreas do país ainda continuarão com condições favoráveis à doença, o que deve implicar na necessidade de adoção de medidas de controle, especialmente no período de novembro a abril (Figuras 1 e 3). Nos meses de menor favorabilidade, maio a outubro (Figuras 2 e 4), as regiões Sudeste e Sul apresentam alterações do clima tornando o período mais úmido e favorável à sigatoka-negra, o que deve significar a necessidade de intensificação das medidas de controle.

Os limites utilizados para caracterizar as condições 
TABELA 1 - Área $\left(1000 \mathrm{~km}^{2}\right)$ de ocorrência favorável à sigatoka-negra da bananeira no Brasil, para o período atual (média de 1961 a 1990) e futuro (2020, 2050 e 2080, para os cenários A2 e B2)

\begin{tabular}{|c|c|c|c|c|c|c|c|}
\hline \multirow{2}{*}{ Meses } & \multirow{2}{*}{ Atual } & \multicolumn{2}{|c|}{2020} & \multicolumn{2}{|c|}{2050} & \multicolumn{2}{|c|}{2080} \\
\hline & & A2 & B2 & A2 & B2 & A2 & B2 \\
\hline Janeiro & 7850,3 & 6789,6 & 6988,1 & 5747,1 & 6269,9 & 4566,1 & 4836,7 \\
\hline Fevereiro & 8042,2 & 7381,1 & 7211,0 & 6123,0 & 6858,9 & 4750,6 & 5409,4 \\
\hline Março & 8286,9 & 7599,5 & 7506,2 & 6869,4 & 7317,2 & 5282,7 & 6031,9 \\
\hline Abril & 7872,0 & 7721,8 & 7593,5 & 6120,3 & 6958,2 & 3051,2 & 3803,4 \\
\hline Maio & 7046,7 & 6341,8 & 6256,8 & 3500,7 & 5132,3 & 1697,9 & 2675,4 \\
\hline Junho & 5850,1 & 4938,8 & 4994,7 & 2816,3 & 3747,2 & 1300,8 & 2015,4 \\
\hline Julho & 4872,3 & 2384,0 & 2394,2 & 1145,7 & 1498,4 & 564,6 & 1007,2 \\
\hline Agosto & 4553,7 & 988,0 & 883,2 & 520,1 & 605,8 & 318,8 & 408,0 \\
\hline Setembro & 4568,6 & 813,3 & 913,9 & 601,8 & 545,5 & 559,4 & 623,9 \\
\hline Outubro & 4723,8 & 1494,0 & 1830,5 & 1190,9 & 1023,0 & 906,7 & 1097,3 \\
\hline Novembro & 6958,3 & 4795,3 & 5015,9 & 2832,3 & 3746,8 & 1599,1 & 1902,7 \\
\hline Dezembro & 7587,1 & 6308,1 & 6351,2 & 4952,7 & 5690,9 & 2832,1 & 4228,8 \\
\hline
\end{tabular}

favoráveis à ocorrência da sigatoka-negra mostraram-se adequados, pois os mapas confeccionados para as condições atuais estão de acordo com os relatos na literatura. Segundo Mouliom-Pefoura et al. (1996), nas temperaturas abaixo de $20^{\circ} \mathrm{C}$ há redução na germinação dos esporos e no crescimento do tubo germinativo, aumentando o período de incubação da doença. Em temperaturas superiores a $30^{\circ} \mathrm{C}$, Romero \& Sutton (1997) relatam que a severidade da doença é reduzida. A umidade relativa alta permite a reprodução assexual do patógeno e a presença de precipitação é particularmente favorável à liberação de ascósporos (Vicente, 1998; Gauhl, 1994). Com a reprodução sexuada, ocorrem recombinações genéticas com o surgimento de células que podem se adaptar às mais diversas condições climáticas e níveis tecnológicos de cultivo. Porém, não estão relatados limites de favorabilidade para a precipitação e, por essa razão, essa variável não foi utilizada neste estudo.

As mudanças climáticas certamente afetarão o desenvolvimento da planta. Nas condições atuais, a banana é cultivada em todas as regiões do país, mas nos cenários futuros, áreas de inaptidão poderão causar alteração da área cultivada comercialmente. Tal situação, porém, pode ser evitada com o melhoramento de cultivares tolerantes às novas condições. Da mesma forma, o patógeno pode sofrer pressão de seleção em favor de linhagens mais adaptadas.

Os modelos climáticos globais disponibilizados pelo IPCC foram pouco utilizados como ferramentas para o estudo dos impactos das mudanças climáticas sobre doenças de plantas. Geralmente, os autores simplificam a metodologia assumindo um valor constante de temperatura ou precipitação a ser somado ou subtraído nos valores médios atuais, independentemente da época do ano e da região geográfica estudada, como por exemplo, Boag et al. (1991) para o estudo do efeito das mudanças climáticas em nematóides fitopatogênicos, Xiphinema e Longidorus, na
Europa; Luo et al. (1995) para simulações com brusone do arroz em países asiáticos; Brasier \& Scott (1994) e Brasier (1996) para a ocorrência de Phytophthora cinnamomi Rands em carvalho (Quercus spp.) no continente europeu; fitopatógenos na Finlândia por Carter et al. (1996) e no Reino Unido, por Clifford et al. (1996). Os modelos climáticos globais provam que tal metodologia não é a mais adequada, pois estão previstas alterações sazonais e espaciais (IPCC, 2001). Trata-se da melhor ferramenta para projetar cenários prováveis de alterações climáticas para o futuro, levando em conta de forma quantitativa o comportamento dos compartimentos climáticos (atmosfera, oceanos, criosfera, vegetação, solos e outros) e de suas interações (NAE, 2005a).

Os modelos climáticos globais apresentam algumas dificuldades para utilização. A baixa resolução temporal e espacial, nos quais as avaliações são baseadas, torna difícil conciliar os cenários previstos com os modelos de respostas biológicas, como o crescimento de plantas ou doenças, que requerem informações diárias ou até mesmo horárias. Um dos grandes desafios é adaptar as exigências dos modelos de processos biológicos às disponibilidades dos modelos de circulação global, com abordagens de longo prazo (Scherm \& van Bruggen, 1994; Scherm, 2004).

Outro importante aspecto é a significativa variação das anomalias de algumas variáveis climáticas entre os modelos. Por exemplo, a precipitação não segue o mesmo padrão de comportamento nos diversos modelos, diferentemente do aumento de temperatura que está projetado em todos eles. Uma alternativa para contornar o problema é o uso da média aritmética dos resultados dos diversos modelos, como realizado no presente trabalho, pois essa operação ameniza tais diferenças, apesar de ser extremamente trabalhosa. Não obstante as limitações, segundo Van Vuuren \& O'Neill (2006), tais cenários continuam a servir como base para a 
avaliação dos impactos das mudanças climáticas globais. Os autores discutiram a consistência dos cenários do IPCC, comparando dados projetados e reais para os últimos anos sobre população, economia, uso de energia e emissões de gases.

Avulnerabilidade da agricultura brasileira com relação à ocorrência de doenças de plantas é um assunto estratégico para o país (NAE, 2005a, b). As mudanças climáticas alterarão o atual cenário dos problemas fitossanitários. Modificações na importância relativa das doenças podem ocorrer em algumas décadas. Os impactos econômicos, sociais e ambientais decorrentes podem ser positivos, negativos ou neutros, pois as mudanças climáticas podem diminuir, aumentar ou não ter efeito sobre os diferentes problemas fitossanitários, em cada região. Para evitar prejuízos, é necessária a avaliação dos principais patossistemas de importância econômica para o país, assim como de importância secundária, pois estes podem vir a causar maiores prejuízos no futuro.

\section{REFERÊNCIAS BIBLIOGRÁFICAS}

BOAG, B., CRAWFORD, J.W. \& NEILSON, R. The effect of potential climatic changes on the geographical distribution of the plant-parasitic nematodes Xiphinema and Longidorus in Europe. Nematologica 37:312-323. 1991.

BRASIER, C.M. Phytophthora cinnamomi and oak decline in southern Europe. Environmental constraints including climate change. Annales des Sciences Forestières 53:347-358. 1996.

BRASIER, C.M. \& SCOTT, J.K. European oak declines and global warming: a theoretical assessment with special reference to the activity of Phytophthora cinnamomi. Bulletin OEPP/EPPO Bulletin 24:221-232. 1994.

CARTER, T.R., SAARIKKO, R.A. \& NIEMI, K.J. Assessing the risks and uncertainties of regional crop potential under a changing climate in Finnland. Agricultural and Food Science in Finnland 5:329-350. 1996

CLIFFORD, B.C., DAVIES, A. \& GRIFFITH, G. UK climate change models to predict crop disease and pest threats. Aspects of Applied Biology 45:269-276. 1996.

GASPAROTTO, L., PEREIRA, J.C.R., HANADA, R.E. \& MONTARROYOS, A.V.V. Sigatoka-negra da bananeira. Manaus. Embrapa Amazônia Ocidental, 2006.

GAUHL, F. Epidemiology and ecology of black sigatoka (Mycosphaerella fijiensis Morlet) on plantain and banana (Musa spp.) in Costa Rica, Central America. Montpellier, França. INIBAP. 1994.

GHINI, R. Mudanças climáticas globais e doenças de plantas. Jaguariúna. Embrapa Meio Ambiente. 2005.

HANSEN, J., SATO, M., RUEDY, R., LO, K., LEA, D.W.
\& MEDINA-ELIZADE, M. Global temperature change. Proceedings of the National Academy Sciences of the USA 103: 14288-14293. 2006.

IPCC. Climate change 2001: the scientific basis: summary for policymakers. Geneva: IPCC, 2001. 20 p. Disponível em: $<$ http:// www.ipcc.ch/pub/spm22-01.pdf>. Acesso em: 26 abr. 2006.

IPCC. The SRES emission scenarios: the IPCC Data Distribution Centre. Disponível em: $<$ http://sedac.ciesin.columbia.edu/ddc/sres/ index.html>. Acesso em: 1 mar. 2006.

IPCC. Climate change 2007: the physical science basis: summary for policymakers. Geneva: IPCC, 2007. 18 p. Disponível em: $<$ http://www.ipcc.ch/SPM2feb07.pdf $>$. Acesso em: 3 maio 2007.

JACOME, L.H. \& SCHUH, W. Effects of leaf wetness duration and temperature of development of black Sigatoka disease on banana infected by Mycosphaerella fijiensis var. difformis. Phytopathology 82:515-520. 1992.

LUO, Y., TEBEEST, D.O., TENG, P.S. \& FABELLAR, N.G. Simulation studies on risk analysis of rice leaf blast epidemics associated with global climate change in several Asian countries. Journal of Biogeography 22:673-678. 1995.

MARENGO, J.A. Mudanças climáticas globais e regionais: avaliação do clima atual do Brasil e projeções de cenários climáticos do futuro. Revista Brasileira de Meteorologia 16:1-18. 2001.

MOULIOM-PEFOURA, A., LASSOUDIÈRE, A., FOKO, J. \& FONTEM, D.A. Comparison of development of Mycosphaerella fijiensis and Mycosphaerella musicola on banana and plantain in various ecological zones in Cameroon. Plant Disease 80:950-954. 1996.

NAE Núcleo de Assuntos Estratégicos da Presidência da República. Volume I: Negociações internacionais sobre a mudança do clima: vulnerabilidade, impactos e adaptação à mudança do clima. Brasília DF. 2005a. (Cadernos NAE, 3).

NAE Núcleo de Assuntos Estratégicos da Presidência da República. Volume II. Mercado de carbono. Brasília DF. 2005b. (Cadernos NAE, 4).

ROMERO, R.A. \& SUTTON, T.B. Reaction of four Musa genotypes at three temperatures to isolates of Mycosphaerella fijiensis from different geographical regions. Plant Disease 81:1139-1142. 1997.

SCHERM, H. Climate change: can we predict the impacts on plant pathology and pest management? Canadian Journal Plant Pathology 26:367-273. 2004.

SCHERM, H. \& van BRUGGEN, A.H.C. Global warming and nonlinear growth: how important are changes in average temperature? Phytopathology 84:1380-1384. 1994.

Van VUUREN, D.P. \& O'NEILL, B.C.O. The consistency of IPCC's SRES scenarios to recent literature and recent projections. Climatic Change 75:9-46. 2006.

VICENTE, L.P. Sigatoka negra (Mycosphaerella fijiensis Morelet) de bananas y plátanos (Musa spp.) en Cuba. Biologia, epidemiologia y manejo integrado de la enfermedad. In: Simposium Internacional sobre sigatoka negra. 1. Manzanillo. Memórias. Manzanillo, México. SAGAR/INIBAP. 1998. pp. 24-42. 